Research Article

\title{
STGC3 inhibits xenograft tumor growth of nasopharyngeal carcinoma cells by altering the expression of proteins associated with apoptosis
}

\author{
Qing-chao Qiu, ${ }^{1,2,}$, Bo Hu ${ }^{1,2,4, *}$, Xiu-pei He ${ }^{5}$, Qiao Luo ${ }^{1}$, Guo-hua Tang ${ }^{1}$, Zhi-feng Long ${ }^{1}$, \\ Zhu-chu Chen ${ }^{3}$ and Xiu-sheng $\mathrm{He}^{1}$ \\ ${ }^{1}$ Cancer Research Institute, University of South China, Hengyang, China. \\ ${ }^{2}$ Department of Medicine, Vanderbilt University, Nashville, USA. \\ ${ }^{3}$ Cancer Research Institute, Central South University, Changsha, China. \\ ${ }^{4}$ Hunan Environment-Biological Polytechnic College, Hengyang, China. \\ ${ }^{5}$ Department of Otolaryngology, The First People's Hospital of Yunnan Province, Kunming, China.
}

\begin{abstract}
STGC3 is a potential tumor suppressor that inhibits the growth of the nasopharyngeal carcinoma cell line CNE2; the expression of this protein is reduced in nasopharyngeal carcinoma compared with normal nasopharyngeal tissue. In this study, we investigated the tumor-suppressing activity of STGC3 in nude mice injected subcutaneously with Tet/pTRE-STGC3/CNE2 cells. STGC3 expression was induced by the intraperitoneal injection of doxycycline (Dox). The volume mean of Tet/pTRE-STGC3/CNE2+Dox xenografts was smaller than that of Tet/pTRE/CNE2+Dox xenografts. In addition, Tet/pTRE-STGC3/CNE2+Dox xenografts showed an increase in the percentage of apoptotic cells, a decrease in Bcl-2 protein expression and an increase in Bax protein expression. A proteomic approach was used to assess the protein expression profile associated with STGC3-mediated apoptosis. Western blotting confirmed the differential up-regulation of prohibitin seen in proteomic analysis. These results indicate that overexpression of STGC3 inhibits xenograft growth in nude mice by enhancing apoptotic cell death through altered expression of apoptosis-related proteins such as Bcl-2, Bax and prohibitin. These data contribute to our understanding of the function of STGC3 in human nasopharyngeal carcinoma and provide new clues for investigating other STGC3-associated tumors.
\end{abstract}

Key words: CNE2 cell line, nasopharyngeal carcinoma, nude mouse, STGC3, Tet-on, two-dimensional electrophoresis.

Received: March 30, 2011; Accepted: August 12, 2011.

\section{Introduction}

Nasopharyngeal carcinoma (NPC) is a squamous-cell carcinoma that occurs in the epithelial lining of the nasopharynx (Wei and Sham, 2005). This neoplasm has an unusual ethnic and geographic distribution, with a high prevalence in southern China, southeastern Asia, northern Africa and Alaska. The annual incidence is as high as 25 cases per 100,000 people in endemic regions, which is about 25-fold higher than in the rest of the world (Yu and Yuan, 2002; Xiong et al., 2004; Bei et al., 2010). This remarkable geographic and racial distribution of NPC indicates that the development of this cancer may be related to genetic and environmental factors (McDermott et al., 2001; Yu and Yuan, 2002).

Send correspondence to Xiu-sheng He. Cancer Research Institute, University of South China, 421001Hengyang, China. E-mail: hexiusheng@ hotmail.com, and Zhuchu Chen. Cancer Research Institute, Central South University, 410078 Changsha, China. E-mail: tcbl@xysm.net.

*These authors contributed equally to this work.
Current evidence suggests that multi-step genetic alterations, including the activation of oncogenes and/or inactivation of tumor suppressor genes, may underlie NPC tumorigenesis (Chen et al., 1999). The tumor suppressor genes p16/MTS1, DLC1, CDH4, IGFBP-6 and DAB2 show homozygous deletions or reduced expression in NPC tissue (Lo et al., 1995; Sun et al., 1995; Kuo et al., 2010; Tong et al., 2010; Du et al., 2011; Low et al., 2011). Over-expression of the $M Y C, R A S$, EZH2, FGF $8 b$ oncogenes has also been detected in some NPC tumors (Porter et al., 1994; Lu et al., 2011; Lui et al., 2011). Furthermore, loss of heterozygosity (LOH) studies have shown that NPC tumors contain deletions on chromosomal arms that include 3p, 9p and 11q (Huang et al., 1994; Hu et al., 1996; Hui et al., 1996).

A common region of allelic loss in NPC involves chromosome 3p21.31-3p21.2 (Zeng et al., 2006a). Using cDNA cloning and RACE procedures, we cloned a novel gene known as STGC3 (GenBank accession no. AY078383) related to NPC from the common minimal de- 
letion region in 3p21 (He et al., 2004). The full-length cDNA of STGC3 contains 1271 bp with a 438 bp open reading frame that encodes a protein with a molecular mass of $\sim 16 \mathrm{kDa}$. STGC3 protein expression in NPC samples is significantly lower than that in normal nasopharyngeal epithelium. STGC3 inhibits the growth of nasopharyngeal carcinoma CNE2 cells in vitro (He et al., 2008), although the tumor suppressor function of this protein is still unclear. In this work, we used a Tet-on regulated mouse model and proteomic analysis to examine the potential tumor suppressor roles of STGC3.

\section{Materials and Methods}

\section{Cell culture}

CNE2 is a poorly differentiated NPC cell line. CNE2/Tet/pTRE and CNE2/Tet/ pTRE/STGC3 cells generated as described elsewhere (He et al., 2008) were cultured in PRIM1640 medium (Invitrogen, USA) supplemented with $10 \%$ fetal calf serum at $37{ }^{\circ} \mathrm{C}$ in a humidified atmosphere of $95 \%$ air - $5 \% \mathrm{CO}_{2}$.

\section{Experiments with nude mice}

Three-week-old male BALB/c nude mice were acquired from the Animal Centre of the Academia Sinica in Shanghai, China. All animal experiments were approved by the National Animal Care and Use Committee of China and done within one week after arrival of the mice.

Twenty-four nude mice were randomly allocated into four groups ( $\mathrm{n}=6 \mathrm{mice} / \mathrm{group}$ ) for treatment with different cells: CNE2+Dox, CNE2/Tet/pTRE+Dox (Vector+Dox), CNE2/Tet/pTRE/STGC3+Dox (STGC3+Dox) and CNE2/Tet/pTRE/STGC3+PBS (STGC3+PBS). The cells were trypsinized, suspended in phosphate-buffered saline (PBS) and $0.2 \mathrm{~mL}$ containing $2 \times 10^{6}$ cells was inoculated under the right armpit of each mouse. The mice were also injected intraperitoneally with either doxycycline (Dox, $20 \mathrm{mg} / \mathrm{kg}$; Roche, Switzerland) or an equal volume of PBS every day for 28 days (Zeng et al., 2006b). Tumor growth was monitored by measuring two perpendicular diameters with vernier calipers every three days, and tumor volume was calculated using the formula $\mathrm{W}^{2} \mathrm{xLx} 0.52$, where $\mathrm{L}$ is the length and $\mathrm{W}$ is the width of the tumor. The mean tumor size was plotted for each group and the ratio of inhibition (expressed in \%) was calculated as (Mean of the vector group - Mean of the transfected group)/(Mean of the vector group) $\times 100$.

\section{Histological examination}

Subcutaneous tumors derived from the cells indicated in the previous section were excised from nude mice under ether anesthesia. The tumors were rinsed twice in normal saline then fixed in $4 \%$ neutral buffered formalin and embedded in paraffin. The sections were mounted on slides, deparaffinized with xylene, rehydrated through graded al- cohols, stained with hematoxylin and eosin (H\&E) and observed by light microscopy.

\section{RNA extraction and RT-PCR}

Total RNA was isolated from xenograft tissues using Trizol reagent (Invitrogen) according to the manufacturer's instructions. Single-stranded cDNA was generated from $2 \mu \mathrm{g}$ of total RNA using a first-strand cDNA synthesis kit (Promega, USA) according to the manufacturer's instructions. STGC3 mRNA was amplified with the following specific primers: forward, 5'-CGG GAT CCA TGG TTC TTG TTT CTT AT-3' and reverse, 5'-GCC CCA AGC TTT AGA GTA ATA AAA GAT TC-3'. PCR was done for 30 cycles, each consisting of denaturation at $94^{\circ} \mathrm{C}$ for $1 \mathrm{~min}$, annealing at $56^{\circ} \mathrm{C}$ for $50 \mathrm{~s}$, extension at $72{ }^{\circ} \mathrm{C}$ for $1 \mathrm{~min}$. $\beta$-Actin (forward primer, $5^{\prime}$ - GGA CCT GAC TGA CTA CCT C-3', reverse primer, 5'- CAT ACT CCT GCT TGC TGA T-3') was used as an endogenous control gene to confirm that equal amounts of sample mRNA were applied to the gels; this gene was also used to normalize the results.

\section{Protein extraction and western blotting}

Xenograft tissues were extracted in lysis buffer $(0.5 \%$ Nonidet P-40/5\% sodium deoxycholate/ $150 \mathrm{mM} \mathrm{NaCl} / 10$ $\mathrm{mM}$ Tris/ $\mathrm{HCl}, \mathrm{pH} 7.5 / 1 \% \mathrm{BSA}$ ) and centrifuged at $4{ }^{\circ} \mathrm{C}$ for $15 \mathrm{~min}$. The samples were separated by SDS-PAGE in 10\% polyacrylamide gels and transferred to PVDF membranes (Millipore, USA). The membranes were incubated with 5\% fat-free milk at room temperature for $1 \mathrm{~h}$, blotted with anti-STGC3 (1:1000) (He et al., 2008), anti-prohibitin (1:2000; Abcam, USA) and anti- $\beta$-actin (1:5000; Santa Cruz Biotechnology, Santa Cruz, CA, USA) antibodies at room temperature for $2 \mathrm{~h}$, washed, and then incubated with peroxidase-conjugate secondary antibody (1:3000; Santa Cruz Biotechnology). Immunoreactive bands were detected by enhanced chemiluminescence (Amersham Biosciences, Sweden).

\section{Immunohistochemistry}

Immunohistochemistry was done in formalin-fixed, paraffin-embedded tissue sections using a standard immunohistochemical technique. The sections were incubated with anti-STGC3 (1:100), anti-Bcl-2 (1:200) and anti-Bax (1:200) antibodies (Santa Cruz Biotechnology) overnight at $4{ }^{\circ} \mathrm{C}$ and then incubated with a biotin-conjugated secondary antibody (1:500; Santa Cruz Biotechnology) for $60 \mathrm{~min}$ at room temperature. Finally, the tissue sections were incubated with diaminobenzidine (DAB, Santa Cruz Biotechnology) until a brown color developed, after which the sections were counterstained with Harris' modified hematoxylin. Sections incubated with PBS instead of the corresponding primary antibody were used as negative controls. The integral optical densities (IOD) were calculated using PIPS-2020 image analysis software. 


\section{Flow cytometry}

Cell suspensions were prepared from xenograft tumor tissue from each of the four groups of mice. After centrifugation and dilution to $1 \times 10^{6}$ cells $/ \mathrm{mL}$, the cells were fixed in $70 \%$ ethanol and stored in fixative at $-20{ }^{\circ} \mathrm{C}$ until analyzed for apoptosis. Fixed cells were washed with PBS and incubated with propidium iodide $(50 \mu \mathrm{g} / \mathrm{mL}$ in PBS) for $30 \mathrm{~min}$ at room temperature in the dark prior to analysis by flow cytometry.

\section{Two-dimensional (2D) electrophoresis}

The tissue samples were suspended in lysis buffer (7 M urea, $2 \mathrm{M}$ thiourea, 2\% NP-40, 1\% Triton X-100, $100 \mathrm{mM}$ DTT, $5 \mathrm{mM}$ PMSF, 4\% CHAPS, $0.5 \mathrm{mM}$ EDTA, $40 \mathrm{mM}$ Tris and $2 \%$ pharmalyte) at $4{ }^{\circ} \mathrm{C}$ for $1 \mathrm{~h}$, and then centrifuged $\left(12,000 \mathrm{rpm}, 30 \mathrm{~min}, 4^{\circ} \mathrm{C}\right)$. The supernatants were collected and the concentration of total proteins was assayed with a 2D-electrophoresis quantification kit (Amersham Biosciences). Isoelectric focusing was done with an IPGphor system using IPG strips (pH 3-10 L, $24 \mathrm{~cm}$; Amersham Biosciences) followed by SDS-PAGE in an Ettan DALT II system (Amersham Biosciences). After SDS-PAGE, analytical 2D gels were used for 2D western blotting and preparative 2D gels were used for protein identification by mass spectrometry following staining with Coomassie blue G-250.

\section{Image analysis}

Coomassie blue-stained 2D gels were scanned with MagicScan software on an Image scanner and analyzed with a PD-Quest system (Bio-Rad Laboratories, USA) according to protocols provided by the manufacturer. The criterion for determining differential protein spot expression was a two-fold increase or decrease in spot intensity between the groups being compared.

\section{MALDI-TOF-MS and database analysis}

Protein spots that showed changes in the intensity of staining were excised from 2D gels and destained with $100 \mathrm{mM} \mathrm{NH}_{4} \mathrm{HCO}_{3}$ in $50 \%$ acetonitrile. The tryptic peptides were extracted with $50 \%$ acetonitrile $/ 2.5 \%$ trifluoroacetic acid, purified on ZipTip C18 columns (Millipore) and mixed with a 4-hydroxy- $\alpha$-cyanocinnamic-acid (HCCA) solution followed by analysis with a Voyager System 4307 MALDI-TOF mass spectrometer (ABI). A trypsin-fragment peak served as the internal standard for mass calibration. A list of the corrected masses was used for peptide mass fingerprinting and proteins were identified by database searches for each peptide mass (http://www.matrixscience.com).

\section{Statistical analysis}

All assays were done at least three times and the results were expressed as the mean \pm SD unless otherwise stated. Statistical analyses were done using Student's $t$-test or one-way analysis of variance (ANOVA) followed by Tukey's multiple comparisons test. Values of $p<0.05$ indicated statistical significance.

\section{Results}

\section{Overexpression of STGC3 inhibits tumor growth of CNE2 cells increase the differentiation in vivo}

We have previously described a CNE2 cell line with inducible STGC3 expression (He et al., 2008). This cell line, referred to as Tet/pTRE-STGC3/CNE2, was originally transfected with the pRevTet-on regulator plasmid and the pRevTRE-STGC3 expression plasmid. Basal (uninduced) STGC3 expression in this cell line is normally undetectable but can be markedly enhanced (induced) by exposure to doxycyclin $(1 \mu \mathrm{g} / \mathrm{mL})$. We speculated that Tet/pTRE$S T G C 3 / \mathrm{CNE} 2$ could also be induced in vivo. To test this hypothesis, Tet/pTRE-STGC3/CNE2 cells were injected subcutaneously into nude mice and STGC3 expression was induced by daily intraperitoneal injections of doxycyclin (20 mg/kg; Figure 1A). RT-PCR, western blotting and immunohistochemical analysis showed that STGC3 was highly expressed in Tet/pTRE-STGC3/CNE2+Dox xenografts (Figure 2).

To determine whether STGC3 inhibits tumor growth, we measured the tumor masses and volumes. Tumors derived from Tet/pTRE-STGC3/CNE2 cells exposed to Dox (STGC3+Dox group) were smaller $(1.74 \pm 0.66 \mathrm{~g})$ than those in the other three groups [CNE2+Dox: $2.86 \pm 0.83 \mathrm{~g}$, Tet/pTRE/CNE2+Dox (Vector+Dox): $2.76 \pm 0.73 \mathrm{~g}$, Tet/pTRE-STGC3/CNE2+PBS (STGC3+PBS): $2.72 \pm$ $0.73 \mathrm{~g}]$. The maximum suppression of tumor growth by Tet/pTRE-STGC3/CNE2+Dox cells compared to Tet/pTRE/CNE2+Dox cells was 37\% (Figure 1A). Timevolume curves for the xenografts showed that tumors in the Tet/pTRE-STGC3/CNE2+Dox group had a slower growth rate than those in the other three groups $(\mathrm{p}<0.05$; Figure 1B).

Previous work with CNE2 cells in vitro showed that STGC3 suppressed anchorage-independent cell growth in soft agar, indicating a tumor-suppressor role for this protein (He et al., 2008). Based on this observation, we used xenograft tissue to assess whether STGC3 affected tumor malignancy in vivo. Histological analysis of sections stained with H\&E showed that xenografts with high STGC3 expression were more differentiated than those in the other three groups. In cells overexpressing STGC3, the nuclei were smaller and more regular in size and shape, the nucleoli were less prominent and the cytoplasm was more abundant (Figure 1C). These results indicate that overexpression of STGC3 decreases the malignancy of CNE2 cells in vivo. 
A

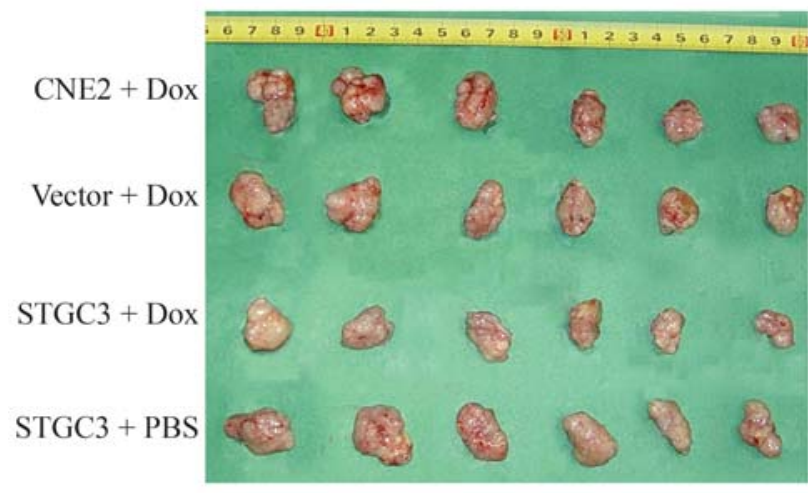

B

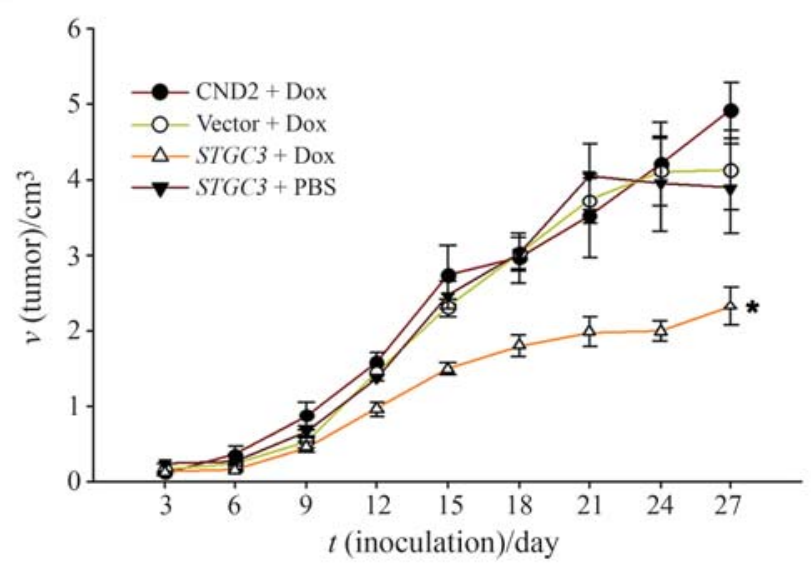

$\mathrm{C}$
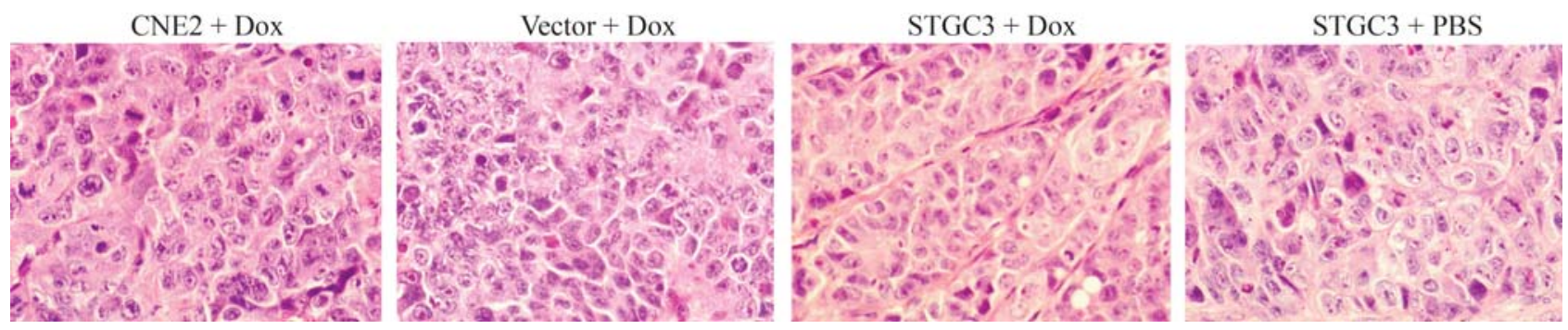

Figure 1 - Over-expression of STGC3 inhibits tumor growth and decreases malignancy in nude mice. (A) Comparison of subcutaneously injected xenografts of nude mice. (B) Time-volume curves for the xenografts. ${ }^{*} \mathrm{p}<0.05$ compared with the Vector+Dox group. (C) STGC3 decreases malignancy in xenograft tissue. Sections were stained with H\&E.

\section{STGC3 increases the percentage of apoptotic cells and affects the expression of Bcl-2 and Bax associated with apoptosis}

To determine the molecular mechanism by which the overexpression of STGC3 causes tumor inhibition in vivo, we used flow cytometry to examine apoptosis in cells from xenograft tumors. Compared to Tet/pTRE/CNE2+DOX xenografts, Tet/pTRE-STGC3/CNE2+Dox xenografts showed a significant increase in the percentage of apoptotic cells ( $11.4 \pm 1.2 \%$ vs. $1.5 \pm 0.6 \%$; $<<0.01$; Figure $3 \mathrm{~A})$. To confirm these results, we examined expression of the apoptosis regulatory protein Bax and the anti-apoptotic protein Bcl-2. In agreement with the flow cytometry results, Bax protein expression was up-regulated and $\mathrm{Bcl}-2$ protein expression was down-regulated in Tet/pTRESTGC3/CNE2+Dox xenografts (Figure 3B,C). The $\mathrm{Bcl}-2 / \mathrm{Bax}$ ratio in the Tet/pTRE-STGC3/CNE2+Dox group was significantly lower than in the other three groups (Table 1 and Figure 3C). These findings suggest that STGC3 increases the percentage of apoptotic cells by down-regulating Bcl-2 expression and up-regulating that of Bax.

\section{Differential expression of proteins associated with overexpression of STGC3}

The nuclear location of STGC3 (Figure 2C) suggests that this protein probably has an important role in regulating nuclear gene transcription. To test this hypothesis, we undertook a comparative proteomic analysis of Tet/pTRE/CNE2+Dox and Tet/pTRE-STGC3/CNE2+Dox xenograft tissues. Figure 4A shows representative examples of the xenograft tissue proteins separated by $2 \mathrm{D}$ electrophoresis. A total of $781 \pm 57(\mathrm{n}=3$ runs) and $749 \pm 46$ ( $\mathrm{n}=3$ runs) protein spots were detected in Tet/pTRE/CNE2+Dox and Tet/pTRE-STGC3/CNE2+Dox xenograft tissues, respectively. Proteins that consistently showed a greater than two-fold change in expression were included in the analysis (Figure 4B).

The differentially expressed protein spots were excised from Coomassie blue-stained gels and subjected to in-gel digestion with trypsin. An aliquot of the supernatant containing tryptic peptides was analyzed by MALDITOF-MS (Figure 4C). Only proteins with a significant score in the Mascot database were identified as being differentially expressed. Figure 4A shows that 11 differentially expressed proteins were identified in 


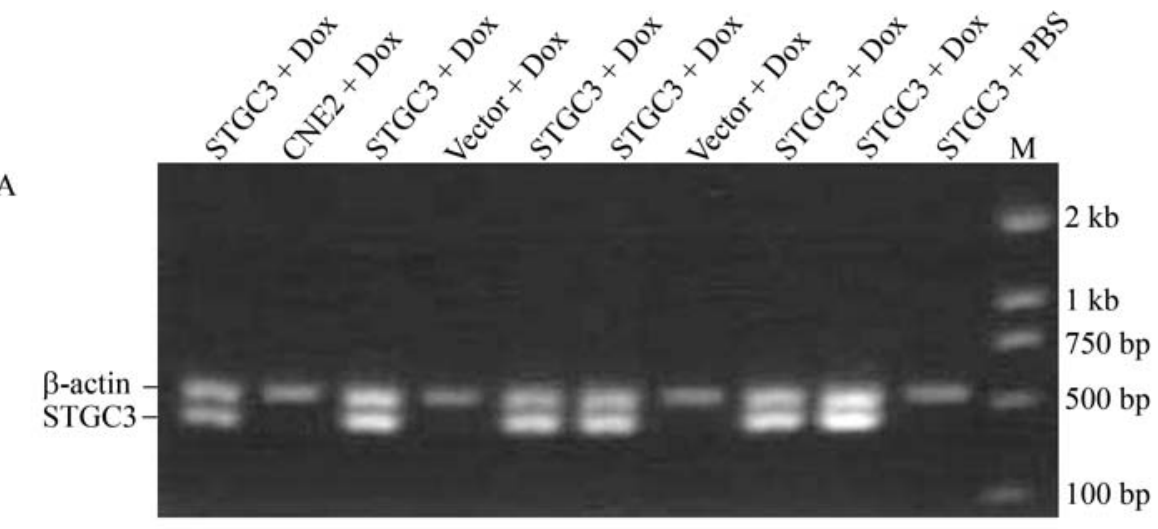

B
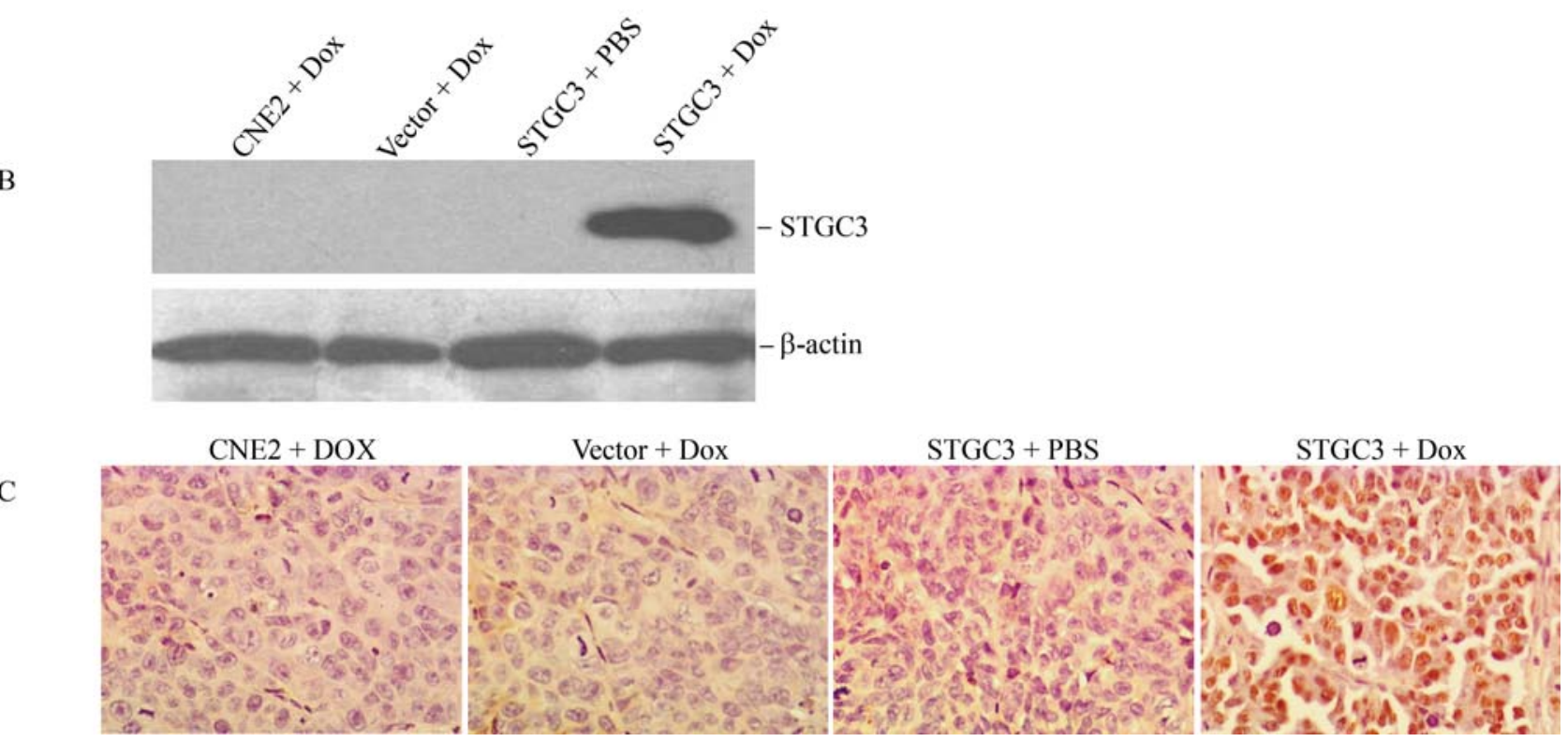

Figure 2 - Dox induces a high level of STGC3 expression in Tet/pTRE-STGC3/CNE2 cells. (A) RT-PCR of STGC3 mRNA expression in xenograft tissue. (B) Detection of STGC3 protein by western blotting with an anti-STGC3 antibody. These results agreed with the RT-PCR data. (C) Immunohistochemical staining for STGC3 in xenograft tissue.

Tet/pTRE/CNE2+Dox and Tet/pTRE-STGC3/CNE2+Dox xenograft tissues. Detailed information for eight of the 11 proteins identified is provided in Table 2; three proteins not included in this table were classified as unknown by the Swiss-Prot website.

Searches of the Swiss-Prot and PubMed databases were used to determine the functions of the identified proteins related to STGC3. Proteins involved in apoptosis were

Table 1 - Effect of STGC3 on Bcl-2 and Bax expression in tumor sections.

\begin{tabular}{lccc}
\hline \multirow{2}{*}{ Groups } & \multicolumn{3}{c}{ Optical density } \\
\cline { 2 - 4 } & $\mathrm{Bcl}-2$ & $\mathrm{Bax}$ & $\mathrm{Bcl}-2 / \mathrm{Bax}$ \\
\hline CNE2+Dox & $48.6 \pm 7.6$ & $16.2 \pm 3.6$ & 3.00 \\
Vector+Dox & $47.4 \pm 6.1$ & $14.2 \pm 1.5$ & 3.34 \\
STGC3+PBS & $49.4 \pm 6.4$ & $15.6 \pm 2.4$ & 3.17 \\
STGC3+Dox & $19.2 \pm 3.7$ & $51.4 \pm 11.5$ & $0.37^{*}$ \\
\hline
\end{tabular}

The values are the mean \pm SD of six sections per group. ${ }^{*} \mathrm{p}<0.05 \mathrm{com}$ pared with the Vector+Dox group. either up-regulated, e.g., prohibitin, or down-regulated, e.g., heat shock 70 protein 8 isoform 2 variant (Powers et al., 2008; Zhu et al., 2010), as were those involved in cell proliferation, e.g., petidyl-prolyl cis-trans isomerase A (up-regulated) and splicing factor arginine/serine-rich 1 (down-regulated) (Colgan et al., 2000; Karni et al., 2007), the cytoskeleton, e.g., cofilin-1 (up-regulated) (Chae et al., 2009), and signal pathways, e.g., serine/threonine-protein kinase WNK1 (down-regulated) and guanine nucleotidebinding protein subunit beta 2-like 1 (up-regulated), when STGC3 was highly expressed in xenograft tissues (Moniz and Jordan, 2010; Zhang et al., 2010).

\section{STGC3 increases the expression of prohibitin, a protein associated with apoptosis}

To confirm the differential expression of the proteins identified by proteomic analysis, western blotting was used to compare the expression of prohibitin in Tet/pTRE$S T G C 3 / \mathrm{CNE} 2+$ Dox xenograft tissue with that in Tet/pTRE/CNE2+Dox xenograft tissue. Figure 4D shows 


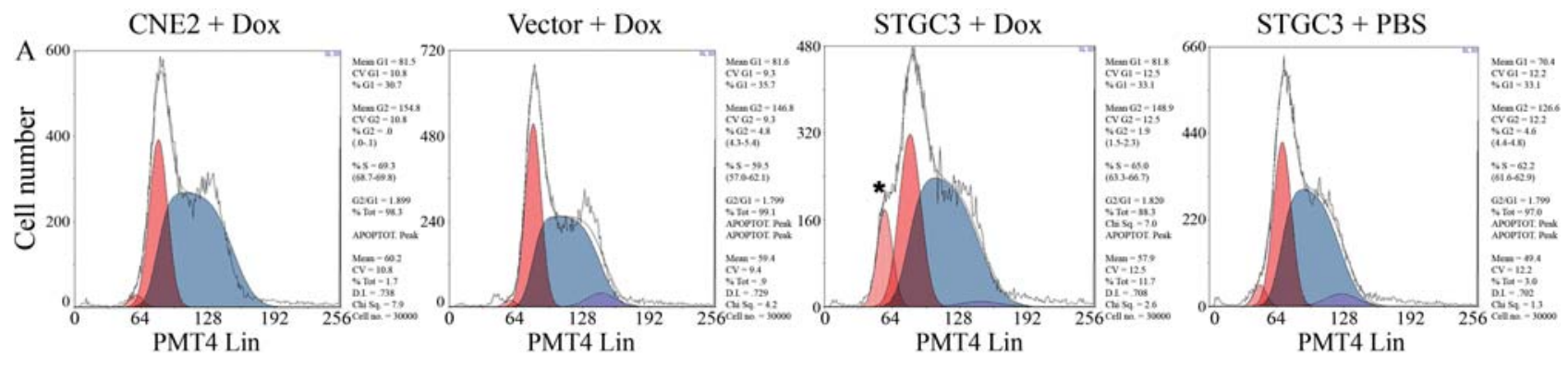

B
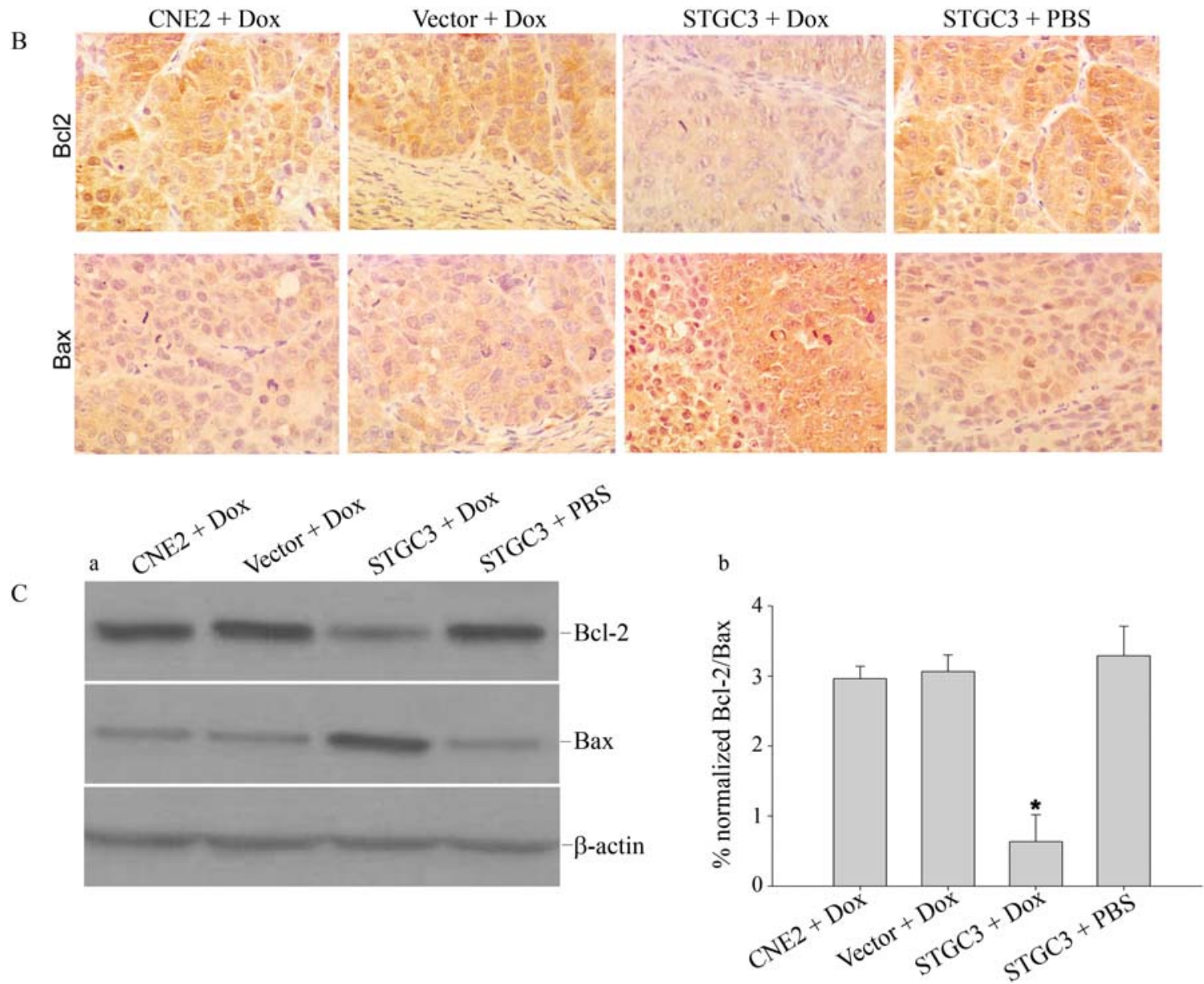

Figure 3 - STGC3 inhibits the tumor growth of CNE2 cells in nude mice by increasing the percentage of apoptotic cells and modulating the expression of the apoptosis-related proteins Bcl-2 and Bax. (A) The percentage of apoptotic cells was increased in Tet/pTRE-STGC3/CNE2+Dox xenografts. *p $<0.01$ compared with the Vector+Dox group. (B) Immunohistochemical staining for Bcl-2 and Bax in xenograft tissue. (C) (a) Bcl-2 and Bax protein expression in xenograft tissue assessed by western blotting. (b) Protein levels normalized to $\beta$-actin. ${ }^{*} \mathrm{p}<0.05$ compared with the Vector+Dox group.

representative western blotting of prohibitin expression in Tet/pTRE/CNE2+Dox and Tet/pTRE-STGC3/CNE2+Dox xenograft tissues. Prohibitin expression in Tet/pTRE$S T G C 3 / \mathrm{CNE} 2+$ Dox tissue was 4.5 -fold greater than in Tet/pTRE/CNE2+Dox tissue. These results were identical to those obtained with $2 \mathrm{D}$ electrophoresis.

\section{Discussion}

In this study, mice received subcutaneous injections of Tet/pTRE-STGC3/CNE2 cells and STGC3 expression was induced by the intraperitoneal injection of Dox. The Tet-on gene system was developed by Gossen and Bujard (1992), who found that gene expression can be easily modulated via the administration of Dox, which is minimally toxic and targets specific genes. As shown here, the intraperitoneal injection of Dox in mice is highly effective in stimulating overexpression of the STGC3 gene.

In vitro, STGC3 inhibits the growth of CNE2 cells and suppresses the anchorage-independent growth of these cells in soft agar, implying a tumor-suppressor role for this 


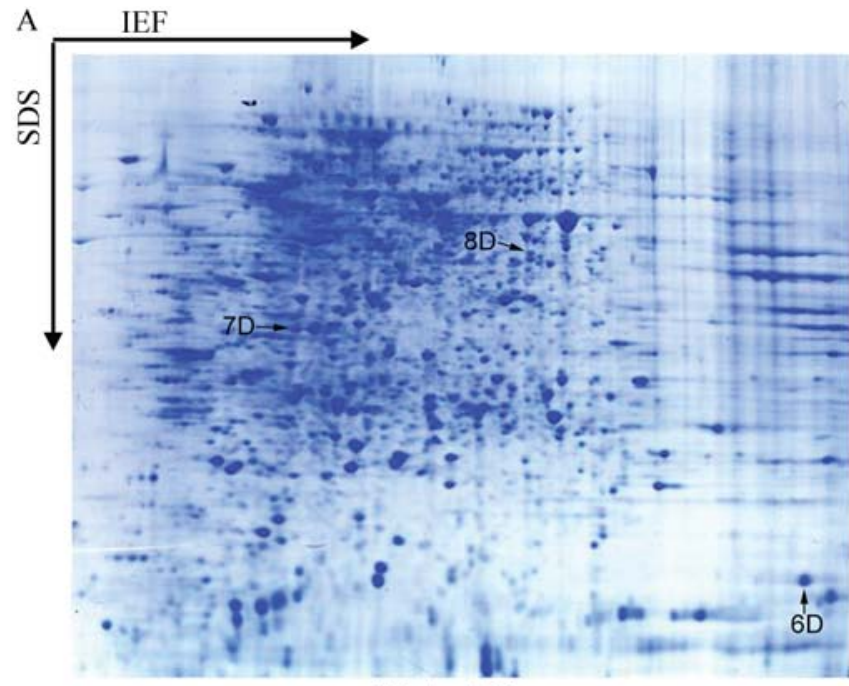

Vector + Dox

B

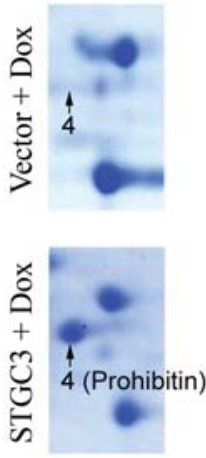

$\mathrm{C}$

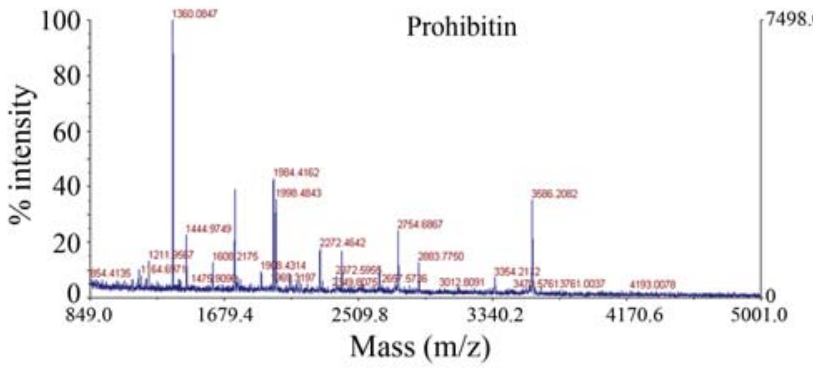

1 MAAKVEESIG KPGLALAVAG GVWNSALYNV DAGHRAVIFD RERGVQDIW

51 GEGTHELIPN VQKPIIFDCR SRPRNVPVIT GSKDLQNVNI TLRILPRPVA

101 SQLPRIFTSI GEDYDERVLP SITYEILKSV VARFDAGELI TQRELVSRQV

151 SDDLTBRAMT FGLILDDVSL THLTFGKERT EAVEARQVAQ QBAERARFW

201 BKAEQQKKAA IISAEGDSKA ABLIANSLAT AGDGLIELRK LEAAEDIAYQ

251 LSRSRNTTYL PAGQSVLLQL PQ

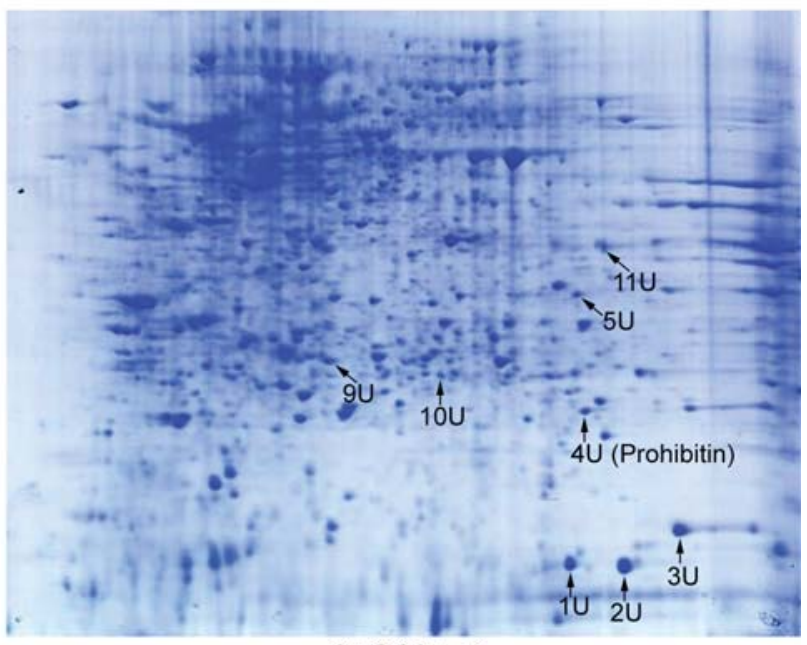

STGC3 + Dox

D
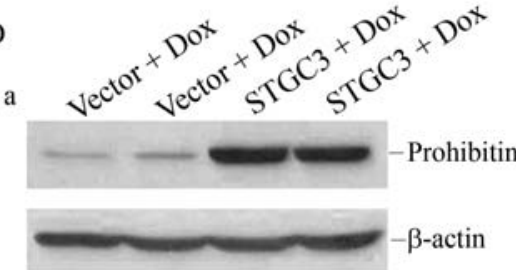

b

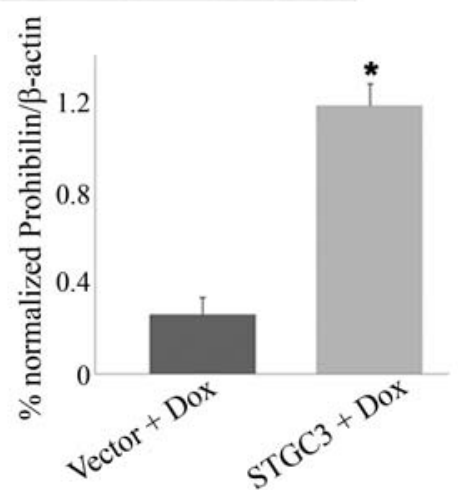

Figure 4 - Comparative proteomic analysis of Tet/pTRE/CNE2+Dox and Tet/pTRE-STGC3/CNE2+Dox xenograft tissues by 2D electrophoresis, mass spectrometry and western blotting. (A) 2D electrophoretic maps of Tet/pTRE/CNE2+Dox and Tet/pTRE-STGC3/CNE2+Dox xenograft tissue proteins. (B) A close-up of the 2D gel showing the marked overexpression of protein spot 4 in Tet/pTRE-STGC3/CNE2+Dox tissue compared with Tet/pTRE/CNE2+Dox tissue. (C) MALDI - TOF mass spectrometric analysis of differential protein spot 4. (D) (a) Western blotting of prohibitin expression in Tet/pTRE/CNE2+Dox and Tet/pTRE-STGC3/CNE2+Dox xenograft tissues. (b) Protein levels normalized to $\beta$-actin. *p $<0.05$ compared with the Vector group.

Table 2 - Up-regulated and down-regulated spots in the Tet/pTRE-STGC3/CNE2+Dox group.

\begin{tabular}{lccccccl}
\hline Spot & $\begin{array}{c}\text { Accession } \\
\text { number }\end{array}$ & $\begin{array}{c}\text { Expressed in } \\
\text { STGC3+Dox }\end{array}$ & $\begin{array}{c}\text { Peptide } \\
\text { matches }\end{array}$ & pI & MW & $\begin{array}{c}\text { Sequence } \\
\text { coverage (\%) }\end{array}$ & Protein identity \\
\hline 1 & P62938 & $\uparrow$ & $9 / 26$ & 7.82 & 13,098 & 58 & Peptidyl-prolyl cis-trans isomerase A \\
3 & P23528 & $\uparrow$ & $5 / 17$ & 8.26 & 18,588 & 53 & Cofilin-1 \\
4 & A46117 & $\uparrow$ & $7 / 28$ & 5.57 & 29,843 & 36 & Prohibitin \\
5 & P63243 & $\uparrow$ & $19 / 38$ & 7.56 & 35,380 & 51 & Guanine nucleotide-binding protein subunit $\beta 2-$ like 1 \\
6 & A46160 & $\downarrow$ & $3 / 9$ & 6.51 & 3,805 & 83 & Serine/threonine-protein kinase WNK1 \\
7 & Q07955 & $\downarrow$ & $15 / 77$ & 7.72 & 22,560 & 70 & Splicing factor arginine/serine-rich 1 \\
8 & Q53HF2 & $\downarrow$ & $14 / 57$ & 5.62 & 53,580 & 65 & Heat shock 70 protein 8 isoform 2 variant \\
11 & P13646 & $\uparrow$ & $10 / 22$ & 8.27 & 22,324 & 67 & Triosephosphate isomerase \\
\hline
\end{tabular}

Note that of the 11 proteins with altered expression, three (proteins 2,9 and 10; not shown in the Table) were identified as 'unknown' in database searches. 'Peptide matches' indicates the number of matches found in database searches compared to the total number of peptides generated by tryptic digestion. 
protein in the development of NPC (He et al., 2008). The results described here indicate that the overexpression of STGC3 inhibits xenograft growth in nude mice, thus confirming the tumor suppressor role of STGC3 in the development of NPC. The increase in the percentage of apoptotic cells when STGC3 was overexpressed indicates that this protein inhibits tumor growth by enhancing apoptosis. This finding was related to the ratio of pro-apoptotic and antiapoptotic proteins in the cell. Bcl-2 is an important suppressor of apoptosis while Bax is a key apoptosis inducer. Cells with a low Bcl-2:Bax ratio are more likely to undergo apoptosis than those with a high Bcl-2:Bax ratio (Xie et al., 1999; Qin et al., 2008). As shown here, Bcl-2 protein expression was down-regulated and Bax protein expression was up-regulated in Tet/pTRE-STGC3/CNE2+Dox xenografts. These data suggest that $\mathrm{STGC} 3$ inhibited the growth of CNE2 cells by altering the ratio of pro-apoptotic and anti-apoptotic proteins.

Proteomic analysis is a powerful tool for the global evaluation of protein expression and has been widely applied in the study of diseases, especially in cancer research. Differential protein expression profiling by 2D electrophoresis is a crucial part of proteomics that can provide accurate, reproducible results on protein expression in two or more biological samples. In the present study, we detected 11 proteins that were differentially expressed in response to the overexpression of STGC3. Among these proteins, we focused on prohibitin, which is involved in the regulation of cell apoptosis. Zhu et al. (2006) reported that prohibitin is an intracellular mediator in the signaling pathway of transforming growth factor $\beta$, a potent apoptosis inducer. The induction of prohibitin is also an indicator of mitochondrial destabilization during apoptosis-related events (Thompson et al., 2001). Many apoptotic signals converge at the level of the mitochondria and release mitochondrial proteins that promote apoptosis. Prohibitin stabilizes mitochondrial membrane proteins such as Bcl-2 and Bax (Manjeshwar et al., 2003). The tumor suppressor role of STGC3 may be associated with the up-regulation of prohibitin and result in increased apoptosis by affecting the mitochondrialassociated proteins Bcl-2 and Bax.

In conclusion, STGC3 inhibits xenograft growth by increasing the percentage of apoptotic cells through alterations in the expression of various genes involved in apoptosis, including the down-regulation of $\mathrm{Bcl}-2$ and upregulation of Bax. STGC3 also affects the expression of genes related to proliferation, the cytoskeleton and cell signaling. The putative tumor suppressor prohibitin was identified as an important candidate protein affected by STGC3. Together, these findings indicate that STGC3 probably has an important role in suppressing NPC tumorgenesis. They also provide a foundation for future investigations of the molecular mechanisms involved in STGC3-mediated tumor suppression.

\section{Acknowledgments}

The work was supported by the National Natural Science Foundation of China (grant no. 81172575), the Specialized Research Fund for the Doctoral Program of Higher Education of China (grant no. 20104324110002), the Key Project of Hunan Province Natural Sciences Foundation of China (grant no. 09JJ3071) and the Key Project of Hunan Province Education Department Foundation of China (grant no. 08A060). We thank Jessica Moore and Joe Fullerton, Vanderbilt University, for critical reading of the manuscript.

\section{References}

Bei JX, Li Y, Jia WH, Feng BJ, Zhou G, Chen LZ, Feng QS, Low HQ, Zhang H, He F, et al. (2010) A genome-wide association study of nasopharyngeal carcinoma identifies three new susceptibility loci. Nat Genet 42:599-603.

Chae JI, Kim J, Woo SM, Han HW, Cho YK, Oh KB, Nam KH and Kang YK (2009) Cytoskeleton-associated proteins are enriched in human embryonic-stem cell-derived neuroectodermal spheres. Proteomics 9:1128-1141.

Chen YJ, Ko JY, Chen PJ, Shu CH, Hsu MT, Tsai SF and Lin CH (1999) Chromosomal aberrations in nasopharyngeal carcinoma analyzed by comparative genomic hybridization. Genes Chromosomes Cancer 25:169-175.

Colgan J, Asmal M and Luban J (2000) Isolation, characterization and targeted disruption of mouse Ppia: Cyclophilin A is not essential for mammalian cell viability. Genomics 68:167178.

Du C, Huang T, Sun D, Mo Y, Feng H, Zhou X, Xiao X, Yu N, Hou B, Huang G, et al. (2011) CDH4 as a novel putative tumor suppressor gene epigenetically silenced by promoter hypermethylation in nasopharyngeal carcinoma. Cancer Lett 309:54-61.

Gossen M and Bujard H (1992) Tight control of gene expression in mammalian cells by tetracycline-responsive promoters. Proc Natl Acad Sci USA 89:5547-5551.

He XS, Xiao ZQ, Chen ZC, Zhao SP, Zhu JH, He ZM, Li YJ, Tian $\mathrm{F}$ and $\mathrm{Yu}$ YH (2004) Molecular cloning and functional analysis of STGC3 - a novel gene on chromosome 3p21. Ai Zheng 23:1110-1115.

He XS, Deng M, Yang S, Xiao ZQ, Luo Q, He ZM, Hu B and Chen ZC (2008) The tumor supressor function of STGC3 and its reduced expression in nasopharyngeal carcinoma. Cell Mol Biol Lett 13:339-352.

Hu LF, Eiriksdottir G, Lebedeva T, Kholodniouk I, Alimov A, Chen F, Luo Y, Zabarovsky ER, Ingvarsson S, Klein G, et al. (1996) Loss of heterozygosity on chromosome arm 3p in nasopharyngeal carcinoma. Genes Chromosomes Cancer 17:118-126

Huang DP, Lo KW, van Hasselt CA, Woo JK, Choi PH, Leung SF, Cheung ST, Cairns P, Sidransky D and Lee JC (1994) A region of homozygous deletion on chromosome 9p21-22 in primary nasopharyngeal carcinoma. Cancer Res 54:40034006.

Hui AB, Lo KW, Leung SF, Choi PH, Fong Y, Lee JC and Huang DP (1996) Loss of heterozygosity on the long arm of chromosome 11 in nasopharyngeal carcinoma. Cancer Res 56:3225-3229. 
Karni R, de Stanchina E, Lowe SW, Sinha R, Mu D and Krainer AR (2007) The gene encoding the splicing factor SF2/ASF is a proto-oncogene. Nat Struct Mol Biol 14:185-193.

Kuo YS, Tang YB, Lu TY, Wu HC and Lin CT (2010) IGFBP-6 plays a role as an oncosuppressor gene in NPC pathogenesis through regulating EGR-1 expression. J Pathol 222:299309 .

Lo KW, Huang DP and Lau KM (1995) p16 gene alterations in nasopharyngeal carcinoma. Cancer Res 55:2039-2043.

Low JS, Tao Q, Ng KM, Goh HK, Shu XS, Woo WL, Ambinder RF, Srivastava G, Shamay M, Chan AT, et al. (2011) A novel isoform of the 8p22 tumor suppressor gene DLC1 suppresses tumor growth and is frequently silenced in multiple common tumors. Oncogene 30:1923-1935.

Lu J, He ML, Wang L, Chen Y, Liu X, Dong Q, Chen YC, Peng Y, Yao KT, Kung HF, et al. (2011) MiR-26a inhibits cell growth and tumorigenesis of nasopharyngeal carcinoma through repression of EZH2. Cancer Res 71:225-233.

Lui VW, Yau DM, Cheung CS, Wong SC, Chan AK, Zhou Q, Wong EY, Lau CP, Lam EK, Hui EP, et al. (2011) FGF8b oncogene mediates proliferation and invasion of EpsteinBarr virus-associated nasopharyngeal carcinoma cells: Implication for viral-mediated FGF8b upregulation. Oncogene 30:1518-1530.

Manjeshwar S, Branam DE, Lerner MR, Brackett DJ and Jupe ER (2003) Tumor suppression by the prohibitin gene 3 '-untranslated region RNA in human breast cancer. Cancer Res 63:5251-5256.

McDermott AL, Dutt SN and Watkinson JC (2001) The aetiology of nasopharyngeal carcinoma. Clin Otolaryngol Allied Sci 26:82-92.

Moniz S and Jordan P (2010) Emerging roles for WNK kinases in cancer. Cell Mol Life Sci 67:1265-1276.

Porter MJ, Field JK, Leung SF, Lo D, Lee JC, Spandidos DA and van Hasselt CA (1994) The detection of the c-myc and ras oncogenes in nasopharyngeal carcinoma by immunohistochemistry. Acta Otolaryngol 114:105-109.

Powers MV, Clarke PA and Workman P (2008) Dual targeting of HSC70 and HSP72 inhibits HSP90 function and induces tumor-specific apoptosis. Cancer Cell 14:250-262.

Qin L, Zhang X, Zhang L, Feng Y, Weng GX, Li MZ, Kong QL, Qian CN, Zeng YX, Zeng MS, et al. (2008) Downregulation of BMI-1 enhances 5-fluorouracil-induced apoptosis in nasopharyngeal carcinoma cells. Biochem Biophys Res Commun 371:531-535.

Sun Y, Hildesheim A, Lanier AE, Cao Y, Yao KT, Raab-Traub N and Yang CS (1995) No point mutation but decreased ex- pression of the p16/MTS1 tumor suppressor gene in nasopharyngeal carcinomas. Oncogene 10:785-788.

Thompson WE, Branch A, Whittaker JA, Lyn D, Zilberstein M, Mayo KE and Thomas K (2001) Characterization of prohibitin in a newly established rat ovarian granulosa cell line. Endocrinology 142:4076-4085.

Tong JH, Ng DC, Chau SL, So KK, Leung PP, Lee TL, Lung RW, Chan MW, Chan AW, Lo KW, et al. (2010) Putative tumour-suppressor gene DAB2 is frequently down regulated by promoter hypermethylation in nasopharyngeal carcinoma. BMC Cancer 10:e253.

Wei WI and Sham JS (2005) Nasopharyngeal carcinoma. Lancet 365:2041-2054.

Xie X, Clausen OP, De Angelis P and Boysen M (1999) The prognostic value of spontaneous apoptosis, Bax, Bcl-2, and p53 in oral squamous cell carcinoma of the tongue. Cancer 86:913-920.

Xiong W, Zeng ZY, Xia JH, Xia K, Shen SR, Li XL, Hu DX, Tan C, Xiang JJ, Zhou J, et al. (2004) A susceptibility locus at chromosome 3p21 linked to familial nasopharyngeal carcinoma. Cancer Res 64:1972-1974.

Yu MC and Yuan JM (2002) Epidemiology of nasopharyngeal carcinoma. Semin Cancer Biol 12:421-429.

Zeng Z, Zhou Y, Zhang W, Li X, Xiong W, Liu H, Fan S, Qian J, Wang L, Li Z, et al. (2006a) Family-based association analysis validates chromosome $3 \mathrm{p} 21$ as a putative nasopharyngeal carcinoma susceptibility locus. Genet Med 8:156-160.

Zeng ZJ, Li ZB, Luo SQ and Hu WX (2006b) Retrovirusmediated tk gene therapy of implanted human breast cancer in nude mice under the regulation of Tet-On. Cancer Gene Ther 13:290-297.

Zhang J, Liu W, Liu J, Xiao W, Liu L, Jiang C, Sun X, Liu P, Zhu Y, Zhang C, et al. (2010) G-protein $\beta 2$ subunit interacts with mitofusin 1 to regulate mitochondrial fusion. Nat Commun $1: 101$.

Zhu B, Fukada K, Zhu H and Kyprianou N (2006) Prohibitin and cofilin are intracellular effectors of transforming growth factor $\beta$ signaling in human prostate cancer cells. Cancer Res 66:8640-8647.

Zhu B, Zhai J, Zhu H and Kyprianou N (2010) Prohibitin regulates TGF- $\beta$ induced apoptosis as a downstream effector of Smad-dependent and -independent signaling. Prostate 70:17-26.

Associate Editor: Emmanuel Dias Neto

License information: This is an open-access article distributed under the terms of the Creative Commons Attribution License, which permits unrestricted use, distribution, and reproduction in any medium, provided the original work is properly cited. 